\title{
A Critical Prospect of Structural Designing of Avian Influenza A/H5N1 Neuraminidase Inhibitors That Evade Tamiflu Resistance
}

\author{
Petar M. Mitrasinovic ${ }^{1,2}$ \\ ${ }^{1}$ Department of Material Science and Chemistry, Faculty of Systems Engineering, Wakayama University, 930 Sakaedani, \\ Wakayama 640-8510, Japan \\ ${ }^{2}$ Department of Natural Sciences, Belgrade Institute of Science and Technology, 11060 Belgrade, Serbia
}

Correspondence should be addressed to Petar M. Mitrasinovic; petar.mitrasinovic@yahoo.com

Received 26 June 2013; Accepted 4 September 2013

Academic Editors: J. Bustamante, M. A. De Souza, K. Sawanyawisuth, and F. Uribe-Salas

Copyright (c) 2013 Petar M. Mitrasinovic. This is an open access article distributed under the Creative Commons Attribution License, which permits unrestricted use, distribution, and reproduction in any medium, provided the original work is properly cited.

\begin{abstract}
The key public health concern is to define the way in which the next influenza pandemic will be controlled. At present, the question of vital importance is: in the absence of a specific avian flu vaccine, could antiviral drugs obstruct a pandemic should the virus spread from birds to humans? The answer to the issue is inevitably related to finding the ways to circumvent Tamiflu resistance that is well documented in the literature. Several remarkable but slightly mutually inconsistent contributions have been recently reported with the aim to facilitate the development of new inhibitors acting on the key target—neuraminidase of avian influenza A/H5N1 virus. Herein, the versatile arguments are critically analyzed and reconciled. Consequently, the most relevant structural basis for the rational design of novel antivirals is elaborated.
\end{abstract}

\section{Introduction}

Rational drug design, as the multidisciplinary process of finding new medications based on knowledge of biological target, is arguably one of the most exciting scientific fields of our time. The major challenges, in general, are the improvement of the existing drugs and the creation of new types of drugs that allow treatment of diseases, such as cancer, AIDS, Parkinsonism, Alzheimer's disease, and influenza, against which no effective drug currently exists. Influenza is known for its enormous economic impact on population health. Of particular concern currently is a lethal avian influenza virus H5N1, known as "bird flu". The highly pathogenic avian influenza is most commonly found in migratory water birds. Water birds are generally resistant to infection caused by avian influenza, but the virus can cause severe disease when it spreads to poultry and other birds. The first cases of humans being infected by H5N1 were detected in Hong Kong in 1997. The key public health concern is to define the way in which the next influenza pandemic will be controlled. Vaccination is not a realistic plan for a rapidly spreading avian influenza pandemic. Vaccines require months to be created, as it is necessary to identify a virus and grow antibodies against it. Besides, vaccine needs to be distributed, which takes time. A vaccine for the avian virus is being developed, but none is yet available. Thus, the question of vital importance is: in the absence of a specific avian flu vaccine, could antiviral drugs obstruct a pandemic should the virus spread from birds to humans [1]?

There are two classes of antiviral drugs for treating influenza: adamantanes-M2 ion channel inhibitors and neuraminidase (NA) inhibitors. Adamantanes (amantadine and rimantadine) are less expensive, more readily available, established as not being effective against most of the isolated avian flu viruses, and their use can cause serious side effects such as seizures [2]. Adamantanes would likely not be useful in an avian pandemic because adamantane-resistant strains frequently emerge, and these strains can be transmitted from person to person [2]. The class of neuraminidase inhibitors consists of the drugs zanamivir and oseltamivir (OTV), having the commercial names Relenza and Tamiflu, 


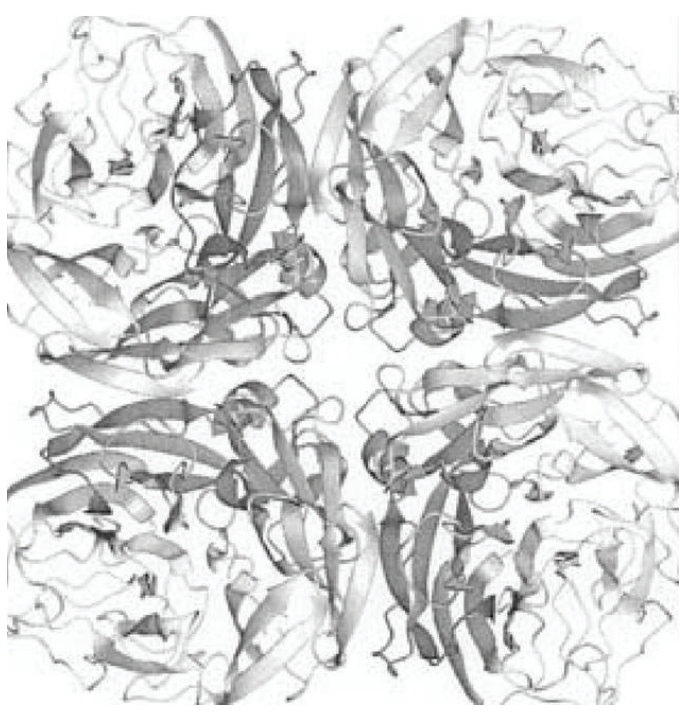

(a)

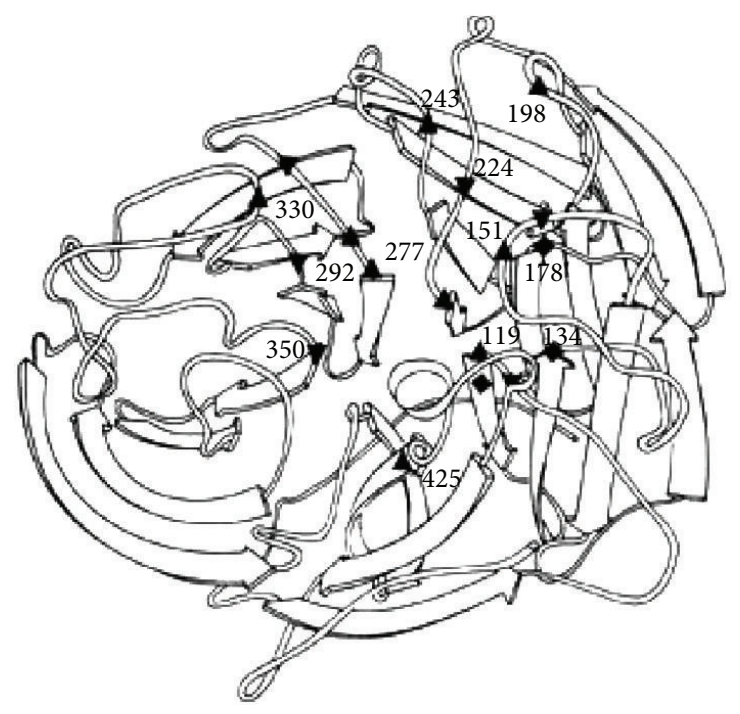

(b)

Figure 1: A typical X-ray structure of neuraminidase tetramer (a). A ribbon diagram of neuraminidase, showing conserved active sites (b) (Education in Chemistry, Issue 2007 March, adapted by permission of the Royal Society of Chemistry) [4].

respectively. If administered early before the virus infects too many cells, these drugs prevent the release of influenza virus from infected cells to healthy ones, and their action is associated with very little toxicity [3]. As neuraminidase enzyme acts at the final stage of infection, NA may be regarded as the key target for developing inhibitors that circumvent oseltamivir resistance [3]. In other words, design of new anti-influenza drugs is essentially design of small molecules that are complementary in shape and charge to NA-biomolecular target to which they are supposed to interact and therefore will bind to it.

Because of different antigenic properties of various glycoprotein molecules, influenza type A NA sequences are divided into two distinct phylogenetic groups, the first group being composed of $\mathrm{N} 1, \mathrm{~N} 4, \mathrm{~N} 5$, and $\mathrm{N} 8$ and the second group being composed of N2, N3, N6, N7, and N9. All influenza NAs are homotetrameric molecules that display fourfold symmetry, with each monomer consisting of six topologically identical four-stranded antiparallel $\beta$-sheets (Figure 1(a)). Under a wide range of experimental conditions, NA is characterized as a rigid crystal with a highly conserved binding site (Figure 1(b)) throughout all the neuraminidase subtypes [4], which is in general a desirable property for the structure-based design of prospective inhibitors. Even though the crystal structures of N1, N4 and N8 of group 1 and the crystal structures of N2 and N9 of group 2 all have the same homotetrameric conformation, they display some group-specific differences in the active site. The main conformational differences between the two groups were detected at the 150-loop (residues 147-152), adjacent to the active site (Figure 2(a)) $[5,6]$. These particular distinctions in the active site architectures were reflected through a $10 \AA \times 5 \AA \times 5 \AA$ 150 -cavity adjacent to the active site in the group 1 NAs that is not present in the group 2 NAs [5]. There is a difference of about $1.5 \AA$ in the positions of the conserved Asp151 side chains, and the carboxylate of the nearby conserved Glu119 in the group 1 NAs points in approximately the opposite direction relative to that in the group 2 NAs, resulting in a width increase of the active site cavity by about $5 \AA$ [5]. The conserved Arg156 with the side chain approximately midway between Asp151 and Glu119 (Figure 2(b)) is located at the base of the 150-cavity (Figure 2(a)) and adopts almost the same position in the group 1 and group 2 NA structures, thus, defining the entrance from the NA active site into the 150-cavity [5]. By examining the crystal structure of the avian influenza neuraminidase in complex with oseltamivir (PDB ID: 2HU0B), known as the "open" conformation of H5N1 NA:OTV complex, the possibility of developing novel inhibitors from the 4-amino group of OTV into the 150cavity was proposed, while the prominent guanidinium side chain of Arg156 was identified as a prospective partner for a salt bridge or hydrogen bond [5]. Despite the experimental proposal [5] and the fact that some structural knowledge of all of the influenza proteins is now known [6], driven by the increased interest due to the threat of the next pandemic, a consistent understanding of the pivotal role of the 150-loop in developing inhibitors that circumvent oseltamivir resistance is not quite clear. Herein, we put forward and reconcile several inconsistencies related to the relevant structural basis as well as elaborate some arguments of vital interest when envisioning more creative strategies for designing new inhibitors of H5N1 NA.

\section{On the Relevant Structural Basis of Designing H5N1 Neuraminidase Inhibitors That Circumvent Tamiflu Resistance}

The "open" conformation of the crystal structure of H5N1 NA in complex with OTV (PDB ID: 2HU0) was used to 


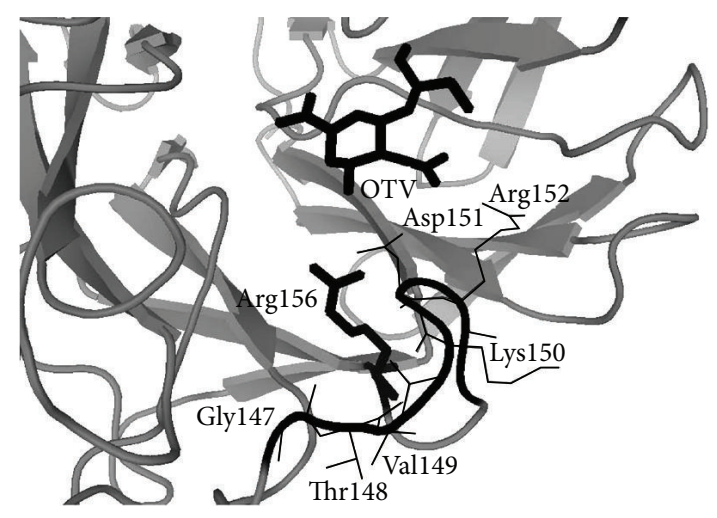

(a)

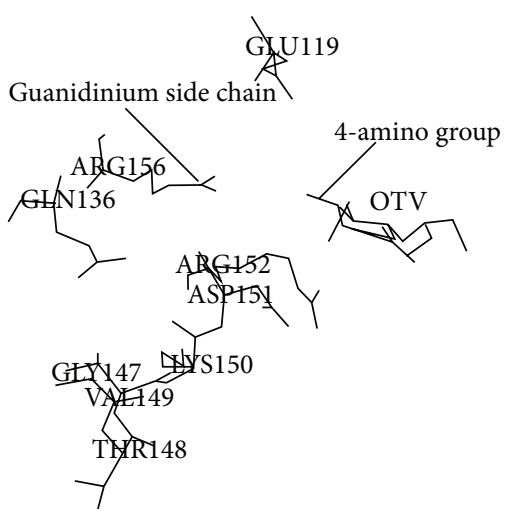

(b)

Figure 2: Experimentally detected 150-loop (Gly147, Thr148, Val149, Lys150, Asp151, and Arg 152) is denoted by bold near the binding site of H5N1 neuraminidase in complex with oseltamivir-OTV (PDB ID: 2HU0) (a). Experimental proposal of exploiting the 150-cavity of H5N1 NA for the design and development of new inhibitors through modifications of the 4-amino group of OTV in order to make a salt bridge or hydrogen bond with the guanidinium side chain of Arg156 [5], (b).

rationalize the molecular mechanism of oseltamivir resistance using molecular docking simulations $[3,7,8]$. It was demonstrated that the 150 -cavity can be exploited for designing novel inhibitors of H5N1 NA $[3,9]$ in the same way as experimentally proposed [5]. The relevance of these observations [3, 7-9] was questioned by pointing out both that an improper initial structure (PDB ID: 2HU0) was used and that a "closed" conformation of the N1:OTV cocrystal structure is more appropriate for such studies [10]. Even though the discrepancies between the theoretical [7] and experimental [11] structures of the His274Tyr N1:OTV protein:ligand complexes were initially observed, the inclusion of protein flexibility, especially in the region of 150-loop, in the molecular docking protocol successfully fixed the glitch $[12,13]$. This quite remarkable rationalization was in agreement with the previously proven considerable flexibility of the residues in the 150-loop of N1 from avian H5N1 [14]. Moreover, the possibility of designing the substantially more effective OTV derivatives than OTV itself was illustrated by both employing the "open" conformation of the N1 NA crystal structure (PDB ID: 2HU0) and taking advantage of the presence of the 150-cavity nearby the N1 NA active site [15]. It was concluded that the "open" form crystal structure of $2 \mathrm{HU} 0$ provides a novel and reliable structural basis for rational drug design against $\mathrm{H} 5 \mathrm{~N} 1$ influenza virus [15], speaking in favor of the previous studies [7-9] being based upon the experimental proposal of Russell et al. [5].

There is no doubt that X-ray crystallography data provide useful information for examining the differences between binding sites in active and inactive conformations or between different neuraminidases. However, the possibility that various conformations in static crystal structures are the consequences of the differences in crystallization conditions, or procedures, suggests that dynamics of neuraminidases may also be explored in order to see into the effects of large protein domain motions and substantial active site rearrangements. In light of this, the conclusion that the "open" conformation of avian influenza neuraminidase (PDB ID: 2HU0) is quite a dependable ground for the structure-based design of new drugs against $\mathrm{H} 5 \mathrm{~N} 1$ influenza virus [15] was substantiated by molecular dynamics simulations $[14,16]$ focusing on the functional behavior of 150-loop in N1 from avian H5N1. Substantially flexible residues in the 150-loop essentially made the avian $\mathrm{N} 1$ protein capable of adopting a large variety of possible configurations in the 150-loop region [14]. Despite the fact that a "closed" 150-loop conformation in the same avian N1 NA was reported under particular experimental conditions [5], atomic-level structural insights based on the molecular dynamics of the neuraminidase enzyme showed that an "open" conformation of the 150-loop is favorable by the avian N1 overall [16]. Interestingly, the crystal structure of the $2009 \mathrm{H} 1 \mathrm{~N} 1$ pandemic virus neuraminidase displayed the lack of 150-cavity in its active site [17], suggesting that the particular 2009 pandemic NA protein was structurally more comparable to the group 2 NAs than to the group 1 NAs. On the basis of alignments of sequences associated with all available NA crystal structures, the position 149 was hypothesized as critical for determining the (open or closed) conformational status of the 150-cavity, suggesting a new paradigm for comprehension of the presence of the 150cavity in group 1 and in group 2 enzymes [16]. However, both oseltamivir (Tamiflu) and zanamivir (Relenza) are equally effective against both phylogenetic groups. Crystal structures of N1 NA in complex with Relenza or Tamiflu revealed that the 150-loop can experience a conformational change upon inhibitor binding in such a way that the binding site of NA from the two groups is essentially identical when bound to an inhibitor [5]. This is the answer to why all of the inhibitors are effective against both phylogenetic groups in spite of their unliganded active sites that display distinct conformations. Of note regarding the design of the next generation of NA inhibitors is that Tamiflu can bind to N1 NA without giving rise to the conformational change of the 150-loop $[5,6]$. The 150 -cavity can thus be targeted by new derivatives of Tamiflu 

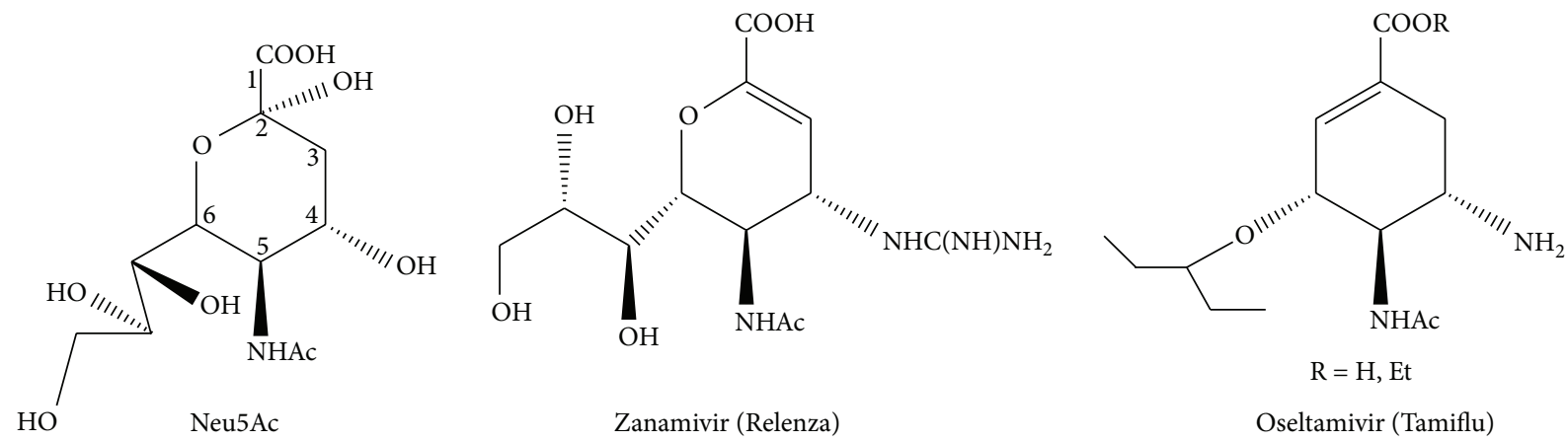

Figure 3: Structural comparison between Relenza and Tamiflu. Sialic acid (N-acetylneuraminic acid or Neu5Ac) is a natural receptor from which the structure of Relenza is directly derived with minimal functional modifications.

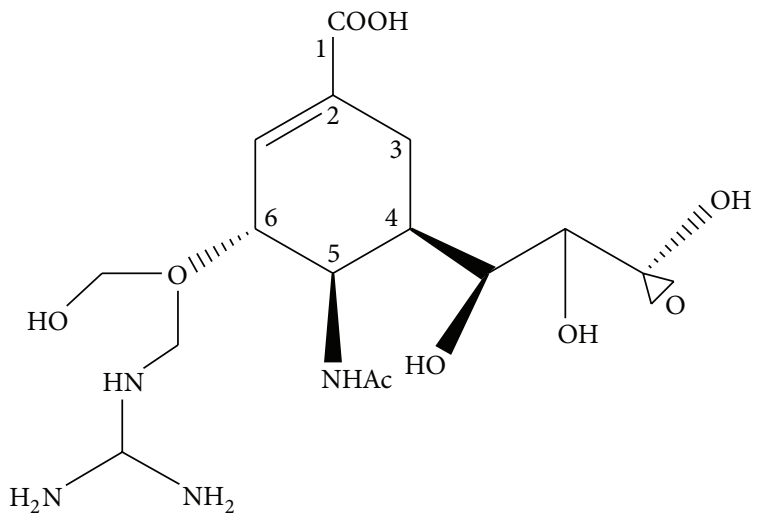

FIGURE 4: The prospective inhibitor [9], designed by simultaneous modifications of both the 4-amino side chain and the C6 bulky hydrophobic side chain of Tamiflu, was shown to concomitantly exploit the experimentally identified location of the 150-cavity in H5N1 NA, as proposed by Russell et al. [5] (Figure 2(b)), and maintain a structural resemblance to sialic acid. Note that the side chain at the C4 position was a slight modification of the C6 side chain of Relenza (Figure 3) and as such was involved in three electrostatic interactions with the guanidinium side chain of Arg156 upon forming the complex with H5N1 NA [9].

or Relenza in order to overcome the virus resistance. To even better understand the structural dynamics of the influenzarelated target receptors in the time ahead, ensemble-based virtual screening methods are being developed in order to enable the use of multiple receptor conformations aimed at: (i) improving the quantitative evaluations of ligand-receptor interactions, (ii) revealing new modes of ligand inhibition, and (iii) developing novel patient-specific therapeutics that are less inclined to drug resistance $[18,19]$.

By using the template structures of a naturally occurring NA inhibitor 2-deoxy-2,3-didehydro-N-acetylneuraminic acid (Neu5Ac2en; Figure 5(a)) in complex with N2 and N9 NAs [20, 21], zanamivir and oseltamivir (Figure 3) were structurally designed $[22,23]$ and approved by the USA Federal Drug Administration (FDA). Since oseltamivir and zanamivir have appeared in the market, clinically relevant development of resistance with unknown side effects has become a critical topic. A clinical experience of more than 8 years has not resulted in providing evidence that the use of zanamivir would result in viable mutants [24], most likely due to a limited use of zanamivir. A substantially wider use of OTV has provided evidence that a viable resistant mutant has emerged [25]. The major structural difference between Tamiflu and Relenza, which influences their activities against avian influenza NA to a great extent, is the presence of a large hydrophobic side chain at the position C6 that is freely rotatable around the $\mathrm{C}-\mathrm{O}$ bond in the structure of Tamiflu, while such a side chain is not present in the structure of Relenza (Figure 3). The development of the resistance to the action of Tamiflu fitting into the NA binding site was understood in light of conformational changes of the amino acids that are responsible for accommodating the Tamiflu's C6 bulky hydrophobic side chain upon ligand binding. In other words, resistance was viewed as the structural consequence of preventing the active site rearrangement due to mutations Arg292Lys, Asn294Ser, and His274Tyr-positions that are near the active site of the enzyme $[11,26]$. Whereas such an active site rearrangement was not needed in the case of Relenza [26], structural studies of the influenza neuraminidase revealed that resistance to Tamiflu is more likely than that to Relenza [26, 27]. The most frequent oseltamivir-resistant mutation in group 1 NAs is His274Tyr [28], while that in group 2 NAs is Asn294Ser which also confers partial resistance to zanamivir $[28,29]$. The possibility of escaping the H5N1 NA mutants may thus be increased by maintaining the clear structural resemblance of a prospective inhibitor candidate to sialic acid (Neu5Ac), a natural receptor from which Relenza is directly derived with minimal functional modifications (Figure 3) [24]. Russell et al. [5] proposed the modification at the C4 position of Tamiflu for developing novel N1-selective inhibitors (Figure 2(b)). This experimental indication [5] was redefined by showing that simultaneous modifications of both the 4-amino side chain and the C6 bulky hydrophobic side chain of Tamiflu are needed and possible in order to concomitantly exploit the experimentally identified location of the 150-cavity and maintain a clear structural resemblance of potential inhibitor candidates to sialic acid (Figure 4) 


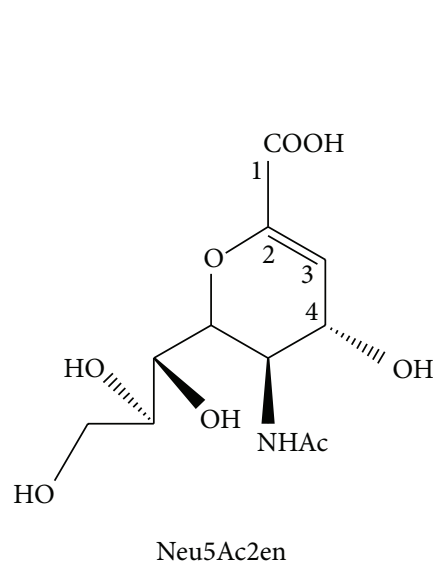

(a)

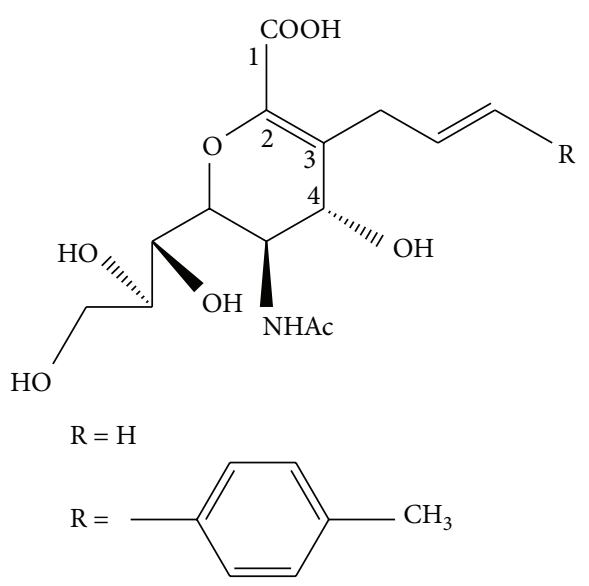

(b)

Figure 5: 2-deoxy-2,3-didehydro-N-acetylneuraminic acid (Neu5Ac2en), (a). The derivatives of Neu5Ac2en designed by introducing allyl and arylallyl substituents at the C3 position of Neu5Ac2en [34], (b). The compounds were shown to occupy 150-cavity and thus selectively target the pandemic $\mathrm{A} / \mathrm{H} 1 \mathrm{~N} 1$ and avian $\mathrm{A} / \mathrm{H} 5 \mathrm{~N} 1$ viruses [34].

[9]. This research progress is well correlated with the work of Wen et al. [30], which demonstrated that a micromolar $\mathrm{IC}_{50}$ can be achieved by extending the moiety at the $\mathrm{C} 4$ position of Relenza to occupy the 150-cavity in the N1 crystal structure. Some other computational studies also attempted to target the 150-cavity in order to design N1-selective inhibitors [19, 31-33]. Rudrawar et al. [34] reported that compounds derived from Neu5Ac2en by introducing allyl and arylallyl substituents at the C3 position of Neu5Ac2en (Figure 5) occupy the 150-cavity and thus selectively target the pandemic $\mathrm{A} / \mathrm{H} 1 \mathrm{~N} 1$ and avian $\mathrm{A} / \mathrm{H} 5 \mathrm{~N} 1$ viruses. In terms of anti-influenza activities being associated with micromolar $\mathrm{IC}_{50}$ values, this study [34] is the first direct evidence of the experimental proposal of Russell et al. [5] that sialic acid derivatives can be more selective for group 1 NAs than for group $2 \mathrm{NAs}$ and equally potent against wild-type NA and the His274Tyr mutant. It means, if a structural design of prospective inhibitors that bind to the highly conserved active site of N1 NA is successful, such a designing strategy is expected to achieve the desired goal of activity against all influenza NA subtypes (N1-N9) and influenza B viruses, as suggested by both NA enzyme inhibition and X-ray crystallography data $[35,36]$.

\section{Conclusion and Future Outlook}

The current progress in the structure-based design of prospective N1 neuraminidase inhibitors indicates the importance of the experimental proposal of Russell et al. [5] for evading oseltamivir resistance. Any synthetic strategy based on the proposal is in agreement with the understanding that the "open" conformation of the crystal structure of the complex of H5N1 NA with oseltamivir (PDB ID: 2HU0) represents a reliable structural basis for the undertaking. However, because distinct conformations in static crystal structures may be the consequences of different crystallization conditions, or procedures, a better understanding of the structural dynamics of the influenza-related target receptors will be needed and provided by emerging ensemblebased virtual screening methods, enabling the use of multiple receptor conformations, revealing new modes of inhibition, and supporting the development of novel patient-specific therapeutics that are less prone to drug resistance.

\section{Acknowledgment}

Part of the work done in Japan was supported by the Japan Society for the Promotion of Science (JSPS).

\section{References}

[1] P. M. Mitrasinovic, "Prefacein," in Global View of the Fight Against Influenza, P. M. Mitrasinovic, Ed., pp. 13-17, Nova Science, New York, NY, USA, 2009.

[2] P. M. Mitrasinovic, "Recent developments and prospects for influenza M2 ion channel inhibitors that circumvent amantadine resistance," Current Bioinformatics, vol. 7, no. 4, pp. 466473, 2012.

[3] P. M. Mitrasinovic, "Advances in the structure-based design of the influenza a neuraminidase inhibitors," Current Drug Targets, vol. 11, no. 3, pp. 315-326, 2010.

[4] G. Laver, "Flu drugs: pathway to discovery," in Global View of the Fight Against Influenza, P. M. Mitrasinovic, Ed., pp. 21-28, Nova Science, New York, NY, USA, 2009.

[5] R. J. Russell, L. F. Haire, D. J. Stevens et al., "The structure of $\mathrm{H} 5 \mathrm{~N} 1$ avian influenza neuraminidase suggests new opportunities for drug design," Nature, vol. 443, no. 7107, pp. 45-49, 2006.

[6] P. S. Kerry and R. J. Russell, “The impact of structural biology on the understanding of the influenza virus and the rational design of antivirals," in Global View of the Fight Against Influenza, P. M. Mitrasinovic, Ed., pp. 155-184, Nova Science Publishers, New York, NY, USA, 2009.

[7] M. L. Mihajlovic and P. M. Mitrasinovic, "Another look at the molecular mechanism of the resistance of $\mathrm{H} 5 \mathrm{~N} 1$ influenza A 
virus neuraminidase (NA) to oseltamivir (OTV)," Biophysical Chemistry, vol. 136, no. 2-3, pp. 152-158, 2008.

[8] M. L. Mihajlovic and P. M. Mitrasinovic, "Applications of the ArgusLab4/AScore protocol in the structure-based binding affinity prediction of various inhibitors of group-1 and group-2 influenza virus neuraminidases (NAs)," Molecular Simulation, vol. 35, no. 4, pp. 311-324, 2009.

[9] P. M. Mitrasinovic, "On the structure-based design of novel inhibitors of H5N1 influenza A virus neuraminidase (NA)," Biophysical Chemistry, vol. 140, no. 1-3, pp. 35-38, 2009.

[10] T. Rungrotmongkol, M. Malaisree, T. Udommaneethanakit, and S. Hannongbua, "Comment on 'Another look at the molecular mechanism of the resistance of H5N1 influenza A virus neuraminidase (NA) to oseltamivir (OTV)"', Biophysical Chemistry, vol. 141, no. 1, pp. 131-132, 2009.

[11] P. J. Collins, L. F. Haire, Y. P. Lin et al., "Crystal structures of oseltamivir-resistant influenza virus neuraminidase mutants," Nature, vol. 453, no. 7199, pp. 1258-1261, 2008.

[12] P. M. Mitrasinovic, "Reply to Comment on 'Another look at the molecular mechanism of the resistance of H5N1 influenza A virus neuraminidase (NA) to oseltamivir (OTV)," Biophysical Chemistry, vol. 141, no. 1, p. 133, 2009.

[13] P. M. Mitrasinovic, "Comment on 'Comment on 'another look at the molecular mechanism of the resistance of $\mathrm{H} 5 \mathrm{~N} 1$ influenza A virus neuraminidase (NA) to oseltamivir (OTV),"' Biophysical Chemistry, vol. 154, no. 2-3, p. 102, 2011.

[14] R. E. Amaro, D. D. L. Minh, L. S. Cheng et al., "Remarkable loop flexibility in avian influenza N1 and its implications for antiviral drug design," Journal of the American Chemical Society, vol. 129, no. 25, pp. 7764-7765, 2007.

[15] S. Q. Wang, X. C. Cheng, W. L. Dong, R. Wang, and K. Chou, "Three new powerful oseltamivir derivatives for inhibiting the neuraminidase of influenza virus," Biochemical and Biophysical Research Communications, vol. 401, no. 2, pp. 188-191, 2010.

[16] R. E. Amaro, R. V. Swift, L. Votapka, W. W. Li, R. C. Walker, and R. M. Bush, "Mechanism of 150-cavity formation in influenza neuraminidase," Nature Communications, vol. 2, no. 1, p. 388 , 2011.

[17] Q. Li, J. Qi, W. Zhang et al., "The 2009 pandemic H1N1 neuraminidase N1 lacks the 150 -cavity in its active site," Nature Structural and Molecular Biology, vol. 17, no. 10, pp. 1266-1268, 2010.

[18] R. E. Amaro and W. W. Li, "Emerging methods for ensemblebased virtual screening," Current Topics in Medicinal Chemistry, vol. 10, no. 1, pp. 3-13, 2010.

[19] L. S. Cheng, R. E. Amaro, D. Xu, W. W. Li, P. W. Arzberger, and J. A. McCammon, "Ensemble-based virtual screening reveals potential novel antiviral compounds for avian influenza neuraminidase," Journal of Medicinal Chemistry, vol. 51, no. 13, pp. 3878-3894, 2008.

[20] J. N. Varghese, J. L. McKimm-Breschkin, J. B. Caldwell, A. A. Kortt, and P. M. Colman, "The structure of the complex between influenza virus neuraminidase and sialic acid, the viral receptor," Proteins, vol. 14, no. 3, pp. 327-332, 1992.

[21] C. L. White, M. N. Janakiraman, W. G. Laver et al., "A sialic acid-derived phosphonate analog inhibits different strains of influenza virus neuraminidase with different efficiencies," Journal of Molecular Biology, vol. 245, no. 5, pp. 623-634, 1995.

[22] M. von Itzstein, W.-Y. Wu, G. B. Kok et al., "Rational design of potent sialidase-based inhibitors of influenza virus replication," Nature, vol. 363, no. 6428, pp. 418-423, 1993.
[23] C. U. Kim, W. Lew, M. A. Williams et al., "Influenza neuraminidase inhibitors possessing a novel hydrophobic interaction in the enzyme active site: design, synthesis, and structural analysis of carbocyclic sialic acid analogues with potent antiinfluenza activity," Journal of the American Chemical Society, vol. 119, no. 4, pp. 681-690, 1997.

[24] M. von Itzstein, "The war against influenza: discovery and development of sialidase inhibitors," Nature Reviews Drug Discovery, vol. 6, no. 12, pp. 967-974, 2007.

[25] Q. M. Le, M. Kiso, K. Someya et al., "Avian flu: isolation of drugresistant H5N1 virus," Nature, vol. 437, no. 7062, p. 1108, 2005.

[26] A. Moscona, "Global transmission of oseltamivir-resistant influenza," New England Journal of Medicine, vol. 360, no. 10, pp. 953-956, 2009.

[27] A. Moscona, "Medical management of influenza infection," Annual Review of Medicine, vol. 59, pp. 397-413, 2008.

[28] V. P. Mishin, F. G. Hayden, and L. V. Gubareva, "Susceptibilities of antiviral-resistant influenza viruses to novel neuraminidase inhibitors," Antimicrobial Agents and Chemotherapy, vol. 49, no. 11, pp. 4515-4520, 2005.

[29] H. L. Yen, L. M. Herlocher, E. Hoffmann et al., "Neuraminidase inhibitor-resistant influenza viruses may differ substantially in fitness and transmissibility," Antimicrobial Agents and Chemotherapy, vol. 49, no. 10, pp. 4075-4084, 2005.

[30] W. H. Wen, S. Y. Wang, K. Tsai et al., "Analogs of zanamivir with modified C4-substituents as the inhibitors against the group-1 neuraminidases of influenza viruses," Bioorganic and Medicinal Chemistry, vol. 18, no. 11, pp. 4074-4084, 2010.

[31] A. T. García-Sosa, S. Siid, and U. Maran, "Design of multibinding-site inhibitors, ligand efficiency, and consensus screening of avian influenza H5N1 wild-type neuraminidase and of the oseltamivir-resistant $\mathrm{H} 274 \mathrm{Y}$ variant," Journal of Chemical Information and Modeling, vol. 48, no. 10, pp. 2074-2080, 2008.

[32] Y. Li, B. Zhou, and R. Wang, "Rational design of Tamiflu derivatives targeting at the open conformation of neuraminidase subtype 1," Journal of Molecular Graphics and Modelling, vol. 28, no. 3, pp. 203-219, 2009.

[33] Q. S. Du, S. Q. Wang, and K. C. Chou, "Analogue inhibitors by modifying oseltamivir based on the crystal neuraminidase structure for treating drug-resistant $\mathrm{H} 5 \mathrm{~N} 1$ virus," Biochemical and Biophysical Research Communications, vol. 362, no. 2, pp. 525-531, 2007.

[34] S. Rudrawar, J. C. Dyason, M. Rameix-Welti et al., "Novel sialic acid derivatives lock open the 150-loop of an influenza A virus group-1 sialidase," Nature Communications, vol. 1, no. 8, p. 113, 2010.

[35] N. A. Roberts and E. A. Govorkova, "The activity of neuraminidase inhibitor oseltamivir against all subtypes of influenza viruses," in Global View of the Fight Against Influenza, P. M. Mitrasinovic, Ed., pp. 93-118, Nova Science, New York, NY, USA, 2009.

[36] E. A. Govorkova, I. A. Leneva, O. G. Goloubeva, K. Bush, and R. G. Webster, "Comparison of efficacies of RWJ-270201, zanamivir, and oseltamivir against H5N1, H9N2, and other avian influenza viruses," Antimicrobial Agents and Chemotherapy, vol. 45, no. 10, pp. 2723-2732, 2001. 

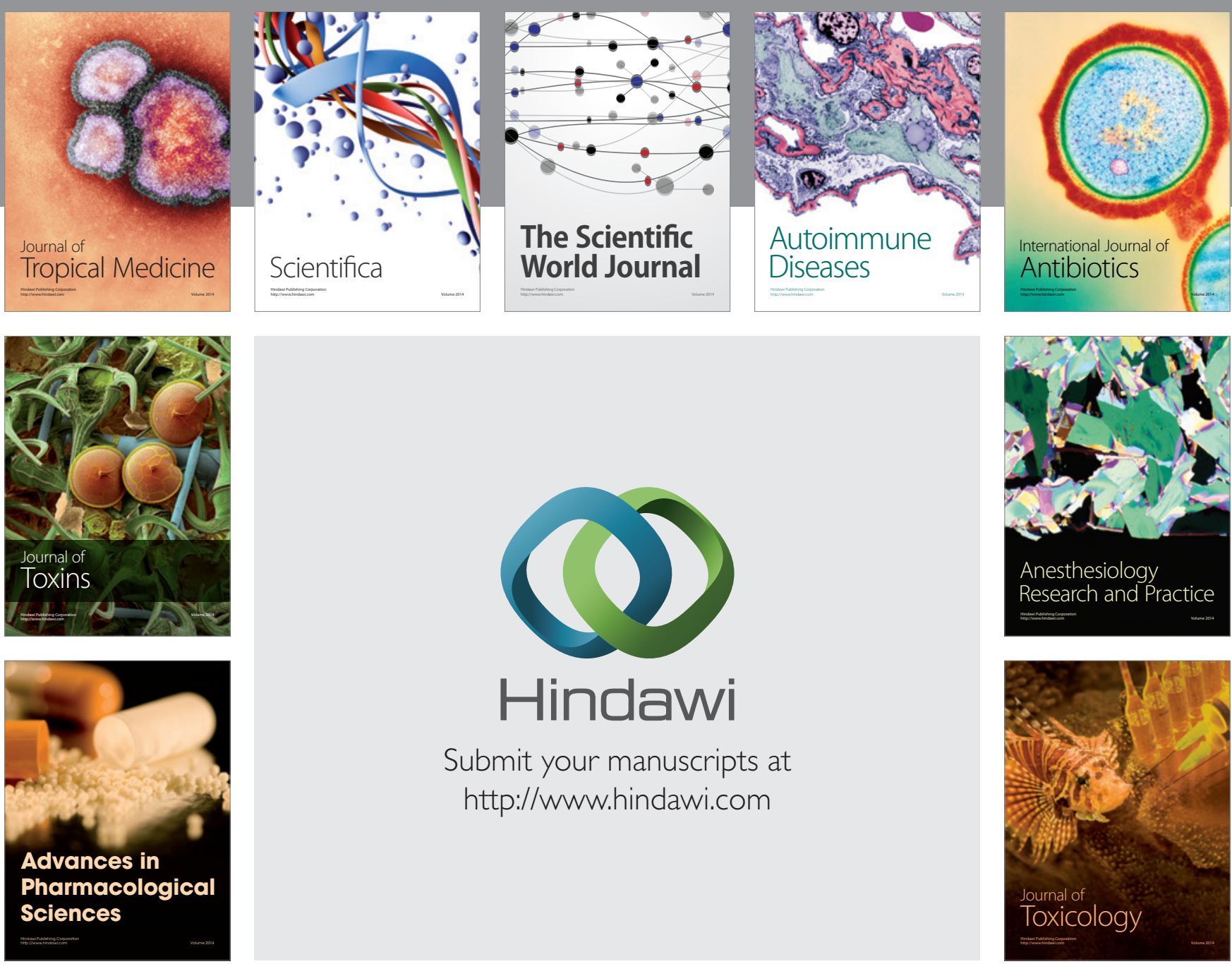

\section{Hindawi}

Submit your manuscripts at

http://www.hindawi.com
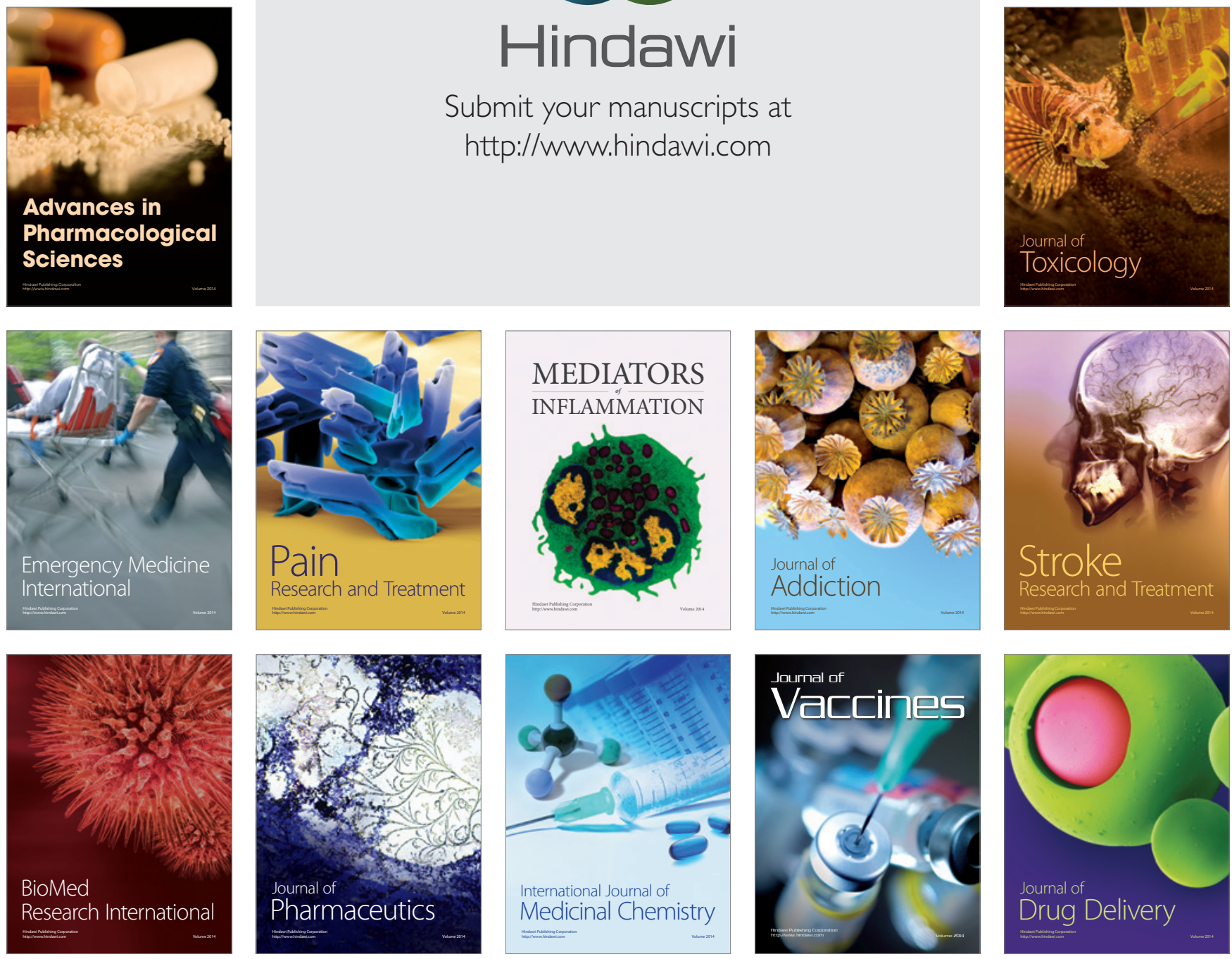